\title{
Comparing Outcomes and Complication of Central Venous Cannulation Using Both Conventional and Ultrasound Guide
}

\author{
Iraj Nazari ${ }^{1 *}$, Masoud Musavi ${ }^{2}$ and Mostafa Alavi ${ }^{3}$ \\ ${ }^{1}$ Assiatant professor in vascular surgery, \\ Ahvaz Jundishapur university of Medical Sciences, Ahvaz, Iran. \\ ${ }^{2}$ Fellowship of Vascular Surgery, Shiraz Medical Sciences University, Shiraz, Iran. \\ ${ }^{3}$ Resident of General Surgery, Ahvaz Jundishapur University of Medical Sciences, Ahvaz, Iran.
}

DOI: http://dx.doi.org/10.13005/bbra/1888

(Received: 10 July 2015; accepted: 03 September 2015)

\begin{abstract}
Central venous cannulation (CVC) is required for management of critically ill and hemodialysis patients which has different complications in conventional procedure. The aim of this study was to compare the outcomes and complication of central venous cannulation using both conventional and ultrasound guide. A randomized controlled trial study of 336 recently hospitalized hemodialysis patients was conducted (168 in intervention group and 168 in control group). CVC was done by an ultrasound machine in intervention group that the needle was inserted perpendicular to the skin under visualization on the US screen while CVC was performed by the conventional landmark approach in the other group. The time for insertion, attempts required, and complications were measured in both groups. Data were analyzed with ANOVA Repeated Measure. In intervention group 22 patients $(13.09 \%)$ required more than one attempt, while in the control group 75 patients $(44.6 \%)$ required more than one attempt. Statistically this difference was significant $(P . V=0.000)$. In the control group, arterial puncture was happened in 10 patients $(5.9 \%)$, and hematoma in 5 patient $(2.9 \%)$, while were 2 patients $(1.1 \%)$ and 1 patients $(0.59 \%)$ in the ultrasound group respectively $(P . V=0.04$ and $P . V=$ 0.05). The results of our study showed that USG approach took lesser time, required lesser attempts, and had lower incidence of complications for cannulation of the internal jugular vein.
\end{abstract}

Key words: Central venous cannulation, Hemodialysis, Conventional, Ultrasound guide.

Central venous cannulation (CVC) is required for management of critically ill and hemodialysis patients. In patients with liver disease and renal failure, CVC is an important intervention for fluid, drugs and concentrated substances such as glucose solution administration and conducting some procedures such as biopsies and measuring venous pressure ${ }^{1}$. Insertion of CVC has both vascular and nonvascular complications, which are decreased in

\footnotetext{
* To whom all correspondence should be addressed. Tel.: 09161135185;

E-mail: irajnazari87@yahoo.com
}

recent years with the introduction of ultrasound guidance (USG) cannulation ${ }^{2-5}$.

USG has different performance such as cause of abdominal distention, DVT and pulmonary status assessment, pericardial tamponade, etc. A common use of ultrasonography in hemodialysis patients is in central venous cannulation, which has been a put blindly using landmark. This conventional cannulation had different complications such as arterial puncture, pneumothorax, hemothorax and air embolism which have been reduced by appearing ultrasonography that the needle can be visualized entering the vein,, ${ }^{7}$ while the conventional method is by direct 
palpation of the artery and puncture with a catheter and followed by threading of the cannula into the vessel which make different complications as mentioned before ${ }^{8}$.

Indications of USG cannulation is for hypotensive patients with difficult palpate of carotid artery, measuring the central venous pressure, administration of inotropes, parenteral nutrition, hemodialysis, etc. Moreover, some of these indications are of emergency that USG CVC can be performed faster than conventional procedure. In some countries, CVC under US guidance is likely to be made compulsory in the near future [9]. This study was designed to investigate the outcomes and complication of central venous cannulation using both conventional and ultrasound guide and compared them in hemodialysis patients.

\section{MATERIALSAND METHODS}

\section{Procedures}

This randomized controlled trial study compared the outcomes and complication of central venous cannulation using both conventional and ultrasound guide in patients needing hemodialysis undergone central venous cannulation with ultrasound guide (study group) to the conventional procedure (control group). An inclusion criterion was all patients who have had indications for central venous cannulation. An exclusion criterion was patients with previous CVC within 15 days, anatomical deformity (such as neck surgery, malignancy and burns on the site), having emergency conditions and bleeding disorders.

In the intervention group, the ultrasound machine SiteRite II (Bard Access, Inc., Salt Lake City, Utah) with 7.5 MHz probe was used. Special jelly as a matching layer for ultrasound was rubbed over the area. The probe was covered with sterile sheath and placed over the anterior neck triangle (between two heads of the sternocleidomastoid muscle (SCM)). The vessels were seen in the transverse section. Internal carotid artery was seen as pulsating, while the internal jugular vein in lateral was nonpulsating which got compressed by pressuring the probe. Then the needle was inserted directed vertically to the skin under visualization on the US screen. After successful aspiration of blood, a J-shaped guide wire was inserted through the hollow needle, then dilator was inserted through the guide wire which was withdrawn and sutured.

In control CVC was performed by the conventional landmark. The patient was in supine position with slight head down with contralateral side position in order to palpate the SCMs and ICA. The ICA was pressed slightly with fingers so that it does not overly the IJV. Then the central line needle was inserted in the lateral of ICA pulsations site. After successful aspiration of blood, rest of the procedure was similar to the intervention group. After the procedure, a chest X-ray was performed for all the patients to rule out a pneumothorax.

\section{Participants}

Participants were recruited between August and December 2014 from Golestan hospitals in Ahvas in Iran. All patients who were hospitalized for central venous cannulation diagnosed by a nephrologist during this time were eligible for participation. From patients 340 cases were selected based on inclusion and exclusion criteria, 4 of them not participate to the study and other 336 cases allocated with a simple random sampling method in 2 groups of intervention and control (168 cases in each groups). The study received ethics approval from the relevant institutional review committees, and all participants gave written informed consent.

\section{Data analysis}

Analyses were performed with statistical package of SPSS (version 20) using descriptive statistics such as mean and standard deviation and analytical statistics such as ANOVA repeated measure.

\section{RESULTS}

The mean age of the patients in intervention group ( 90 males, 78 females) was 54.8 \pm 7.6 years and in control group (87 males, 81 females) was $49.9 \pm 10.6$ years. Table 1 shows comparison of demographic findings of two groups.

The mean time to successful aspiration of venous blood after completion prep of skin in intervention group was 132.52 secs, while in control group was 169.2 secs which was statistically significant $(\mathrm{P} . \mathrm{V}=0.000)$. In intervention group 22 patients (13.09\%) required more than one attempt, while in the control group 75 patients (44.6\%) 
Table 1. Comparison of demographic variables in two groups

\begin{tabular}{|c|c|c|c|c|}
\hline \multicolumn{2}{|l|}{ GroupsVariables } & Intervention & Control & $\mathrm{P}$-value \\
\hline \multicolumn{2}{|l|}{ Age: Mean( \pm SD) } & $54.8(7.6)$ & $49.9(10.6)$ & 0.073 \\
\hline \multirow[t]{2}{*}{ Sex: Number (\%) } & Male & $90(53.5)$ & $87(51.7)$ & 0.74 \\
\hline & Female & $78(46.4)$ & $81(48.2)$ & \\
\hline \multicolumn{2}{|c|}{ Time of renal failure : Mean( \pm SD) (year) } & $9.23(4.5)$ & $10(5.1)$ & 0.701 \\
\hline
\end{tabular}

Table 2. Comparison of outcomes and complications in both studied groups

\begin{tabular}{llccc}
\hline Groups Variables Mean $( \pm$ SD , n) & Intervention & Control & P-value \\
\hline $\begin{array}{l}\text { Time from skin prep to successful } \\
\text { aspiration (in seconds) } \\
\text { Attempts for }\end{array}$ & No. of patients required & $132.52( \pm 15.1,168)$ & $169.2( \pm 16.21,168)$ & 0.000 \\
& more than one attempt $(\%)$ & $22(13.09)$ & $75(44.6)$ & 0.000 \\
successful cannulation & Mean No. of attempt & $1.4( \pm 0.42,168)$ & $1.98( \pm 0.61,168)$ & 0.03 \\
Complications & Arterial puncture & $2(1.1)$ & $10(5.9)$ & 0.04 \\
& Hemothorax & 0 & $1(0.59)$ & 0.970 \\
& Pneumothorax & 0 & $2(1.1)$ & 0.87 \\
& Hematoma & $1(0.59)$ & $5(2.9)$ & 0.05 \\
& Sudden death & 0 & 0 & N.S \\
\hline
\end{tabular}

required more than one attempt. Statistically this difference was significant $(P . V=0.000)$. An average of 1.4 attempts per cannulation was required for intervention group, whereas for control group average 1.98 attempts were required (Table 2 ).

In the control group, arterial puncture was happened in 10 patients (5.9\%), and hematoma in 5 patient (2.9\%), while were 2 patients (1.1\%) and 1 patients $(0.59 \%)$ in the ultrasound group respectively $(P . V=0.04$ and $P . V=0.05)$ (Table 2$)$.

\section{DISCUSSION}

Side effects of CVC are not rare and on the other hand they can be serious problems with poor prognosis in some cases. Recently the use of ultrasound for CVC has been studied to reduce the complications which are dependent to operator and the availability of the equipment ${ }^{10}$. The use of ultrasonography with professional operator decrease the number of complications and attempts compared with the routine method (landmark method). In some studies, it was indicated that the complications of CVC insertion are increased in three or more attempts compared with a single attempt ${ }^{11}$. In our study, it was found that in intervention group 22 patients (13.09\%) required more than one attempt, while in the control group 75 patients (44.6\%) required more than one attempt which increase the complications as mentioned before. Ishii et al. performed a study looking at infants and small children requiring radial artery cannulation and They showed that success rates after a single attempt were significantly higher in the US group $(76.3 \%)$ than those in the conventional group (35.6\%) $(\mathrm{p}<0.001)^{12}$.

On the other hand Ganesh et al. found no statistically significant differences between the US-guided cannulation and palpation technique groups in the time to successful cannulation, total number of attempts, number of successful cannulations during the first attempt or number of cannulae used for catheterization ${ }^{13}$.

Moreover our study showed that the complications of conventional method was more in totally compared with ultrasound group but just two of complications show significant differences between two groups (arterial puncture and hematoma). Several randomized controlled trials have showed the value of US in arterial catheter insertion compared with conventional procedure. Shiver and colleagues demonstrated that a first-pass 
success rate was $87 \%$ in the US group compared with $50 \%$ in the conventional group. Moreover they showed that US method was associated with a $43 \%$ reduction in the development of hematoma at the insertion site ${ }^{14}$. Levin et al. indicated a first-pass success rate was $62 \%$ in US group compared with $34 \%$ in control group ${ }^{15}$. In the study performed by Ankit Agarwal et al. demonstrated that arterial puncture happened in four patients (10\%), and pneumothorax in one patient (2.5\%) in conventional procedure, while there were no such complications in the ultrasound group ${ }^{16}$.

While Tada et al showed that US guidance offered no additional benefit in cases where the radial arterial pulse was palpable ${ }^{17}$. This difference in results of mentioned study and our study was the sample size which was 336 in our study and 166 in Tada's study. Moreover, the procedures are different that we used central venous, whereas they study on the peripheral artery line insertion.

On the other hand in some literatures indicated that disadvantage associated with USGguided CVC, is procedure-related increased incidence of infection, which can be reduced by the use of a two-operator technique with sterile self-adhesive plastic and povidone iodine solution ${ }^{18}$.

\section{CONCLUSION}

The results of our study showed that USG approach took lesser time, required lesser attempts, and had lower incidence of complications for cannulation of the internal jugular vein. Regular use of USG for CVC will benefit for patients required CVC for most of time especially hemodialysis patients. It would be a costly investment in a developing country like Iran, one must keep in mind that use of USG is a prudent approach as USGguided CVC is easier, quicker, and safer than landmark approach.

\section{ACKNOWLEDGMENTS}

We gratefully acknowledge the dedicated efforts of the investigators, the coordinators, and the volunteer patients who participated in this study.

\section{REFERENCES}

1. Doerfler ME, Kaufman B, Goldenberg AS. Central venous catheter placement in patients with disorders of hemostasis. Chest. 1996; 110: 185-8.

2. Denys BG, Uretsky BF, Reddy PS. Ultrasoundassisted cannulation of the internal jugular vein. A prospective comparison to the external landmark-guided technique. Circulation. 1993; 87: 1557-62.

3. Sznajder JI, Zveibil FR, Bitterman H, Weiner P, Bursztein S. Central vein catheterization. Failure and complication rates by three percutaneous approaches. Arch Intern Med. 1986;146: 25961.

4. Oguzkurt L, Tercan F, Kara G, Torun D, Kizilkilic O, Yildirim T. US-guided placement of temporary internal jugular vein catheters: Immediate technical success and complications in normal and high-risk patients. Eur $J$ Radiol. 2005; 55: 125-9.

5. Tercan F, Ozkan U, Oguzkurt L. US-guided placement of central vein catheters in patients with disorders of hemostasis. Eur J Radiol. 2008; 65: 253-6.

6. Julie L, Martin D, Andrew F. Real time ultrasonographically guided internal jugular vein catheterization in the emergency department increases success rates and reduces complications: A randomized, prospective study. Ann Emerg Med. 2006; 48:540-7.

7. Gilbert TB, Seneff MG, Becker RB. Facilitation of internal jugular venous cannulation using an audio-guided doppler ultrasound vascular access device: Results from a prospective, dual-center, randomized, crossover clinical study. Crit Care Med. 1995; 23: 60-5.

8. Ohara U, Nakayama S, Furukawa H, Satoh Y, Suzuki H, Yanai H. Use of a wired-guided cannula for radial arterial cannulation. J Anesth. 2007; 2(1): 83-85.

9. Jefferson P, Ogbue MN, Hamilton KE, Ball DR. A survey of the use of portable ultrasound for central vein cannulation on critical care units in the UK. Anaesthesia. 2002;57:365-8.

10. Bailey PL, Glance LG, Eaton MP, Parshall B, McIntosh S. A survey of the use of ultrasound during central venous catheterization. Anesth Analg. 2007;104:491-7.

11. Thompson C, Frank J, Beecker J, Yeung M, Woo MY, et al. Effectiveness of a novel training program for emergency medicine residents in ultrasound guided insertion of central venous catheters. CJEM.2009;11:343-48.

12. Ishii S, Shime N, Shibasaki M, Sawa T. 
Ultrasound-guided radial artery catheterization in infants and small children. Pediatr Crit Care Med. 2013;14(5):471-473.

13. Ganesh AG, Kaye R, Cahill AM, et al. Evaluation of ultrasound-guided radial artery cannulation in children. Pediatr Crit Care Med. 2009; 10(1): 45-48.

14. Shiver S, Blaivas M, Lyon M. A prospective comparison of ultrasound-guided and blindly placed radial arterial catheters. Acad Emerg Med. 2006; 13(12): 1257-1279.

15. Levin PD, Sheinin O, Gozal Y. Use of ultrasound guidance in the insertion of radial artery catheters. Crit Care Med. 2003; 31(2): 481-484.

16. Ankit Agarwal, Dinesh K. Singh, Anil P. Singh. Ultrasonography: A novel approach to central venous cannulation. Indian J Crit Care Med. 2009; 13(4): 213-216.

17. Tada T, Amagasa S, Horikawa H. Absence of efficacy of ultrasonic two-way Doppler flow detector in routine percutaneous arterial cannulation. J Anesth. 2003;17(3): 206-207.

18. Subhan I, Jain A, Joshi M. Asepsis in ultrasound guided central venous access: A new technique. Ann Emerg Med. 2009; 54: S100. 